\title{
LAMP kit for diagnosis of non-falciparum malaria in Plasmodium ovale infected patients
}

\author{
Juan Cuadros ${ }^{1 *}\left(\mathbb{D}\right.$, Alexandra Martin Ramírez², Iveth J. González ${ }^{3}$, Xavier C. Ding 3 , Ramon Perez Tanoira ${ }^{4,5}$, \\ Gerardo Rojo-Marcos ${ }^{6}$, Peña Gómez-Herruz ${ }^{1}$ and Jose Miguel Rubio ${ }^{7}$
}

\begin{abstract}
Background: Microscopy and rapid diagnosis tests have a limited sensitivity in diagnosis of malaria by Plasmodium ovale. The LAMP kit (LoopAMP ${ }^{\circledR}$ ) can be used in the field without special equipment and could have an important role in malaria control programmes in endemic areas and for malaria diagnosis in returned travellers. The performance of the Pan primer of the kit in detecting malaria by P. ovale was compared with the results of standard $\mathrm{nPCR}$ in samples of patients returning from $P$. ovale endemic areas.
\end{abstract}

Methods: Plasmodium ovale positive samples (29, tested by PCR and/or microscopy) and malaria negative specimens (398, tested by microscopy and PCR) were collected in different hospitals of Europe from June 2014 to March 2016 and frozen at $-20^{\circ} \mathrm{C}$. Boil and spin method was used to extract DNA from all samples and amplification was performed with LoopAMP ${ }^{\circledR}$ MALARIA kit (Eiken Chemical, Japan) in an automated turbidimeter (Eiken 500). The results of LAMP read by turbidimetry and with the naked eye were compared.

Results: The kit showed a sensitivity of $100 \%$ and a specificity of $97.24 \%$ with positive and negative predictive values of 72.5 and $100 \%$, respectively. Naked eyed readings were in accordance with turbidimetry readings (sensitivity, $92.5 \%$, specificity, $98.96 \%$ and positive and negative predictive values, respectively, 90.24 and $99.22 \%$ ). The limit of detection of LAMP assay for P. ovale was between 0.8 and 2 parasites/ $\mu$ l.

Conclusions: The Pan primer of the Malaria kit LoopAMP ${ }^{\circledR}$ can detect $P$. ovale at very low-levels and showed a predictive negative value of $100 \%$. This tool can be useful in malaria control and elimination programmes and in returned travellers from P. ovale endemic areas. Naked eye readings are equivalent to automated turbidimeter readings in specimens obtained with EDTA.

Keywords: Malaria, LAMP, Loop-mediated isothermal amplification, Plasmodium ovale

\section{Background}

Malaria incidence has recently been declining globally, although in many African countries it is still one of the top health problems. The efforts to control and eradicate malaria will still need a strong diagnostic capacity, which allows any parasitaemic patient to be detected and treated sooner. LAMP is a simple molecular diagnostic method based on the principle of isothermal amplification, which does not require special equipment or special distribution in laboratories, and provide results in $60 \mathrm{~min}$

\footnotetext{
*Correspondence: jcuadros48@gmail.com; juan.cuadros@uah.es 1 Department of Clinical Microbiology and Parasitology, Hospital Príncipe de Asturias, 28805 Alcalá de Henares, Madrid, Spain

Full list of author information is available at the end of the article
}

[1]. Different clinical studies have validated this rapid molecular test in the field with a performance similar to conventional PCR $[2,3]$. Additional advantages of LAMP are its tolerance to inhibitory substances present in blood samples (such as haemoglobin and immunoglobulin) [4] and the possibility of being used also on small amounts of blood on filter papers. Furthermore, it could be combined with simple techniques such as microwave DNA extraction [5] in basic laboratories in low resource settings. On the other hand, the growth in international travel and migration has increased the incidence of imported malaria cases in developed countries. In Spain, submicroscopic malaria is common in sub-Saharan migrants (up to $35.5 \%$ in one series [6]) and Plasmodium ovale 
infection may represent up to $8 \%$ of imported malaria cases, as shown in some published series of mainly West African patients [7]. Therefore, sensitive molecular tools are needed both for malaria control programmes and for detecting with certainty malaria imported cases in patients returning from $P$. ovale endemic areas.

LAMP has showed excellent sensitivity and specificity in diagnosing Plasmodium falciparum and Plasmodium vivax malaria, but there is little information about the performance of the test for other species, such as Plasmodium malariae, P. ovale and Plasmodium knowlesi. Although no specific $P$. ovale LAMP primers is commercially available, the PAN primer of the LoopAMP ${ }^{\circledR}$ test could be used for initial detection of $P$. ovale infections.

The primary objective of this study was to determine the diagnostic validity of LoopAMP ${ }^{\circledR}$ in comparison to microscopy and conventional multiplex nested PCR (nPCR) in the diagnosis of $P$. ovale infections in archived clinical specimens. Secondary objectives were to compare the naked eye reading of the amplification products with the automated reading by turbidimetry as well as to determine the specificity of the P. falciparum LoopAmp ${ }^{\circledR}$ primer in specimens of patients infected with $P$. ovale.

\section{Methods}

The study protocol was previously approved by the Ethics Committee of the Hospital Príncipe de Asturias, Madrid, Spain. Signed informed consents were obtained from all individuals with $P$. ovale malaria from whom blood samples were collected.

\section{Sample collection}

The sample size of positive $P$. ovale samples was limited by the availability of positive specimens $(n=30 ; P$. ovale wallikeri 13 , P. ovale curtisi 8 , unknown subspecies 9). These samples were collected with EDTA from venous blood in patients with confirmed $P$. ovale malaria by microscopy and/or PCR and then frozen at $-20{ }^{\circ} \mathrm{C}$. No mixed infections were included in this study. The positive specimens were obtained in different European hospitals for routine testing between June 2014 and March 2016 in the context of an ongoing prospective clinical study on $P$. ovale infections which is being developed in Spain and Europe [7]. The number of negative specimens to be tested was established in 411, according to the prevalence of $P$. ovale imported malaria in our hospital (HUPA) in relation with other species $(6.8 \%, 18$ out of 263 total malaria cases attended in the period 2007-2015) and the total population studied (1.7\% of 1007 patients with suspected malaria in the same period). These negative specimens were obtained from patients coming from West and Central Africa who attended Spanish hospitals with a febrile syndrome and tested negative in thick films, RDTs and PCR multiplex for malaria [8] and were preserved in the clinical specimens Archive of the Microbiology Department of Hospital Príncipe de Asturias and in the Bio Bank of the Malaria laboratory (C.0001392) of the Spanish National Center for Microbiology of Majadahonda.

\section{Sample processing}

The boil and spin method [9] was used for extracting the DNA from frozen whole blood of all patients in a sample preparation area separated from the amplification area. Briefly, an aliquot of $60 \mu \mathrm{l}$ of whole blood of each patient was transferred to the extraction tube and mixed with $60 \mu \mathrm{l}$ of extraction buffer $(400 \mathrm{mM} \mathrm{NaCl}, 40 \mathrm{mM}$ Tris $\mathrm{pH}$ $6.5,0.4 \%$ SDS) by vortex for $10 \mathrm{~s}$. Extraction tubes containing the samples were placed in a hot-block (Techne DRI-Block DB) at $95^{\circ} \mathrm{C}$ for $5 \mathrm{~min}$, and were subsequently centrifuged at $10,000 \mathrm{~g}$ for $3 \mathrm{~min}$ (Jouan MR23). Finally, $30 \mu \mathrm{l}$ of clear supernatant were transferred to a dilution tube with $345 \mu \mathrm{l}$ of sterile water (DNA samples were then stored at $-20{ }^{\circ} \mathrm{C}$ for a maximal period of 2 months for testing).

\section{LAMP reaction and reading}

The commercial Pan and P. falciparum LoopAMP ${ }^{\circledR}$ kits (Eiken Chemical Co., ref. LMP561 and LMC562, respectively) were tested with extracted DNA of blood samples collected in EDTA and kept frozen at $-20{ }^{\circ} \mathrm{C}$. Since EDTA can produce unspecific fluorescence under UV light, the readings of the amplifications products were performed by automated turbidimetry with a turbidimeter (Eiken 500) and with the naked eye [10]. Due to the possibility of DNA degradation with time, the archived $P$. ovale PCR positive specimens testing negative for Loo$\mathrm{pAMP}^{\circledR}$ were rechecked by conventional PCR to ascertain DNA viability.

The Pan specific primers detect a target mitochondrial DNA sequence common to all the Plasmodium species infecting humans. From the dilution tubes, $30 \mu \mathrm{l}$ of extracted DNA was added in a reaction tube for Pan or P. falciparum and then were shaken following the kit instructions for mixing and dissolving. For each batch of 16 reactions, one amplification positive control and one amplification negative control were included alongside (14 samples and 2 controls). The Eiken 500 Turbidimeter was configured with the settings for malaria reaction (amplification at $65{ }^{\circ} \mathrm{C}$ for $40 \mathrm{~min}$ and enzyme inactivation at $80{ }^{\circ} \mathrm{C}$ for $5 \mathrm{~min}$ ). All the amplifications reactions were also read in a blind manner with the naked eye for the purpose of comparison with the standard turbidimetry. 


\section{Multiplex nested PCR}

All samples were tested previously by a validated multiplex nPCR [8] and repeated, as well by duplicate, in case of discordance with the LAMP result. The primer used target the small subunit (SSU) rRNA gene and involves a sequence of two multiplex PCR amplifications. The first reaction amplifies Plasmodium ssrDNA from blood samples infected with malaria and includes a positive reaction control, amplification of the Human ssrDNA gene, which indicates whether the reaction is working properly or not. The second reaction enables the identification of the four human malaria species (P. vivax, $P$. falciparum, $P$. ovale and $P$. malariae) for the fragment size of the product amplified, mixed infection yield the corresponding fragments for species involved.

\section{Limit of detection of the LAMP technique}

The limit of detection (LoD) of the Pan primer LAMP assay for $P$. ovale was determined using two whole blood specimens with a known parasitaemia of $P$. ovale by microscopy (8111 and 2015 parasites/ $\mu \mathrm{l})$. Briefly, each sample was serially tenfold diluted down to 0.08 and 0.02 parasites $/ \mu$ l, respectively, with blood from a Spanish patient negative for $\mathrm{VIH}, \mathrm{VHB}$, and $\mathrm{VHC}$ and no story of travelling to endemic malaria areas. DNA was extracted from each dilution by the boil and spin method and then tested by LAMP twice.

\section{Statistical methods}

Sensitivity, specificity, positive and negative predictive values with $95 \%$ confidence intervals (CI) were calculated using the nPCR as the reference test by means of EPI Dat (3.1) (2006) Sergas [Software] [11].

\section{Results}

A total of 427 clinical samples (29 P. ovale positive and 398 malaria negative) were used to evaluate the performance of the LoopAMP malaria kit for the detection of $P$. ovale parasites in clinical samples. Of the 30 patients with $P$. ovale malaria previously confirmed by $\mathrm{nPCR}$, one sample was excluded due to lack of amplification by LAMP as well as by nPCR when retested. This is possibly due to degradation of DNA over time or an error in initial diagnosis. The other 29 samples were positive with the Pan probe of LAMP, obtaining a sensitivity of $100 \%$ (29/29; CI $95 \%$ : 98.3-100\%). Of the 398 negatives controls remaining after excluding those samples with degraded DNA and abnormal curves (see Additional file 1), 11 samples were positive with LAMP ( $\mathrm{nPCR}$ was repeated in these samples and was negative) obtaining a specificity of $97.24 \%$ (387/398; CI 95\%: 95.5-99\%) compared with nPCR. Positive and negative predictive values were, respectively, $72.5 \%$ (CI 95\%: 57.4-87.6\%) and 100\% (CI 95\%: 99.9-100\%) (Table 1).
Table 1 Comparison of the Pan primer LoopAmp ${ }^{\circledR}$ with nested PCR $(n=427)$

\begin{tabular}{llc}
\hline & LAMP positive & LAMP negative \\
\hline PCR positive & 29 & 0 \\
PCR negative & 11 & 387
\end{tabular}

Sensitivity 100\% (29/29; Cl 95\%: 98.3-100\%); Specificity: 97.24\% (387/398; Cl 95\%: $95.5-99 \%)$. PPV and NPV, respectively, $72.5 \%$ (Cl 95\%: $57.4-87.6 \%)$ and 100\% (Cl 95\%: 99.9-100\%)

Table 2 Comparison of reaction readings with the naked eye or the turbidimeter Eiken $\mathbf{5 0 0}$

\begin{tabular}{lcc}
\hline & NE positive & NE negative \\
\hline Turbidimetry Pos & 37 & 3 \\
Turbidimetry Neg & 4 & 381 \\
\hline
\end{tabular}

Sensitivity, 92.5\% [37/40; Cl 95\%: 83.09-100\%]; Specificity, 98.96\% [380/384 Cl 95\%: 98.81-100\%]; PPV and NPV, respectively, 90.24\% [Cl 95\%: 79.94-100\%] and 99.22\% [Cl 95\%: 98.20-100\%]

The comparison with naked eye (NE) readings was possible in 384 LAMP negative specimens (of 387 negative controls only 3 were indeterminate by NE reading) and 40 turbidimetry positive specimens (29 positive controls and 11 false positive results). All in all, turbidimetry and NE were in good agreement, with only 4 false positive and 3 false negative results by NE: Sensitivity, 92.5\% [37/40; CI 95\%: 83.09-100\%]; Specificity, 98.96\% [380/384 CI 95\%: 98.81-100\%]; positive and negative predictive values, respectively, 90.24\% [CI 95\%: 79.94-100\%] and 99.22\% [CI 95\%: 98.20-100\%] (Table 2).

Plasmodium falciparum LAMP reactions were found to be negative in 29 out of 29 confirmed $P$. ovale samples. One $P$. ovale sample positive by $P$. falciparum LAMP was excluded as it was found to be also positive for $P$. falciparum by nPCR. In this testing session, 8 specimens produced initially an abnormal curve (see Additional file 2) which was considered negative. In all these specimens LAMP test was repeated and a flat curve negative test was confirmed.

The LoD of the Pan LAMP assay for the detection of $P$. ovale parasites were determined to be at 0.8 parasites $/ \mu \mathrm{l}$ in one of the two samples evaluated and 2 parasites $/ \mu$ in the other one. Duplicate testing was performed with the same results and similar Tt in the replicates (Additional file 3). Then, it can be assumed a LOD of 2 parasites/ $\mu \mathrm{l}$, as it was the lowest value at which all replicates were positive, with a proportion of $2 / 4$ samples detected at lower values ( 0.8 parasites/ $\mu \mathrm{l})$.

In the study, an episode of contamination during one of the amplification sessions was detected (unexpected occurrence of false positive results in some tubes in the 
same day, $\mathrm{n}=14)$. No apparent breaking of the protocols nor incidents during pipetting procedures or leaking could be detected. To solve the problem, before the next amplification session, all working surfaces, equipments and reagents were decontaminated with sodium hypochlorite, a new working space for the amplification reaction was established, and the DNA of all false positive samples was re-extracted from archived specimens. The LAMP test was repeated with all these second DNA samples and the number of false positive results was reduced to 5 .

\section{Discussion}

Malaria control and eradication programmes worldwide require reliable tools for detecting very low parasite densities in asymptomatic patients, where microscopy and RDTs can produce false negative results, as it is the case in areas of low transmission where submicroscopic malaria can be a key factor in the control programmes $[12,13]$. Non $P$. falciparum infections have lower parasite densities than $P$. falciparum infections [12] and the sensitivity of RDTs in $P$. ovale and $P$. malariae infections can be very low [14]. On the other hand, for travellers returning from $P$. ovale endemic countries, an affordable molecular rapid test which no requires special equipment with a very high negative predictive value for all common species of malaria could be very useful.

In this study, it is demonstrated the accurate and sensitive detection of $P$. ovale parasites using Plasmodium genus-specific LAMP assay. This is the hitherto largest reported study about LAMP in $P$. ovale malaria.

The obtained results confirm the high sensitivity and specificity of LAMP (100 and 97.2\%, respectively) for detecting $P$. ovale infected patients with the PAN primer when comparing to nested PCR, as other studies showed previously with $P$. falciparum (99 and 93\%) in Thailand [15], all five human malaria species in Malaysia (100 and $100 \%$ ) [16], and in a remote clinic in Uganda (89.5 and $95.9 \%$ ) [1], suggesting that malaria LAMP is a useful molecular tool for detection of low-density malaria infections, including malaria caused by $P$. ovale. Of the 427 clinical samples tested, only 11 resulted in discordant results between LAMP and multiplex nested-PCR. All these discordant results were false positives compared to nPCR results. However, another explanation for these results classified as "false-positive" could be that they are actually true positive of very-low parasitaemia that were undetected by the nPCR reference standard, as has been previously reported [16].

Naked-eye readings showed a very good correspondence with the results obtained by turbidimetry. This result confirm that LAMP can be used in field settings when there is no turbidimeter available, the blood is extracted with EDTA anticoagulant and the UV fluorescence reading method cannot be used [17].

The limit of detection of $P$. ovale malaria was determined between 2 and 0.8 parasites/ $\mu$ l of blood, even more sensitive than the results obtained in previous studies about LoDs for $P$. ovale, which were performed with malachite green-LAMP or with ultraviolet (UV) light as reading methods ( 3 and 10 , respectively) $[16,18]$.

The occurrence of a contamination episode could have had an impact in the specificity results, if it had remained undetected. Contamination could be due to the semiautomated nature of the test and the high sensitivity of the LAMP technique as has been also documented in previous studies $[1,15]$. In our case, LAMP reaction tubes were tightly closed and never opened after amplification and read out was done by turbidimetry and with the naked eye. Most of the samples were frozen in a different clinical centre, from where the study was completed, and then the DNA was extracted and frozen in the laboratory again until LAMP was done. All these manipulations, which are not common in clinical settings, could have increased the chances of contamination.

No false-negative results were detected and this suggests that the Pan malaria LoopAmp ${ }^{\circledR}$ can be used with confidence in malaria eradication or control programmes where $P$. ovale is endemic, although the kit does not include a set of $P$. ovale specific primers and further testing as nested specific PCR would be necessary for final species identification. However, the specific identification of $P$. ovale in the field would not be crucial, as patients could be treated with chloroquine for a non-falciparum malaria and the final identification could be made later in a reference center.

\section{Conclusion}

The Pan primer of the Malaria kit LoopAMP ${ }^{\circledR}$ can detect $P$. ovale at very low-levels and it showed a predictive negative value of $100 \%$. This tool can be useful in malaria control and elimination programmes and in returned travellers from $P$. ovale endemic areas. Naked eye readings are equivalent to automated turbidimeter readings in specimens obtained with EDTA. The relative simplicity of the LAMP procedure and the low infrastructure costs open a range of opportunities by bringing molecular-level parasite detection and capacity of using malaria LAMP in field settings $[1,17]$.

\section{Additional files}

Additional file 1. Raw data of the study comparing turbidimeter and naked eyed readings.

Additional file 2. Abnormal curves obtained by turbidimetry.

Additional file 3. Results of the LoD study. 


\section{Abbreviations}

LAMP: loop-mediated isothermal amplification; nPCR: nested polymerase chain reaction; EDTA: ethylenediamine tetraacetic acid.

\section{Authors' contributions}

JC, JR, IG and GR conceptualized the study, JC, AM and JR designed the experiments, XD and RP provided training and supervision for the procedures, AM, $J C$ and PG performed the experiments. All authors contributed to the writing of the final version of the manuscript. All authors read and approved the final manuscript.

\section{Author details}

1 Department of Clinical Microbiology and Parasitology, Hospital Príncipe de Asturias, 28805 Alcalá de Henares, Madrid, Spain. ${ }^{2}$ Department of Clinical Microbiology and Parasitology, Hospital de la Princesa, Madrid, Spain.

${ }^{3}$ Foundation for Innovative New Diagnostics (FIND), Geneva, Switzerland.

${ }^{4}$ Department of Medicine and Laboratory, Gambo Rural General Hospital, Kore, West-Arsi, Gambo, Ethiopia. ${ }^{5}$ Department of Otorhinolaryngology-Head and Neck Surgery, University of Helsinki and Helsinki University Hospital, Helsinki, Finland. ${ }^{6}$ Department of Internal Medicine, Hospital Príncipe de Asturias, Madrid, Spain. ${ }^{7}$ Malaria and Emergent Protozoa Laboratory, National Center for Microbiology of Majadahonda, Madrid, Spain.

\section{Acknowledgements}

We would like to thank the Spanish P ovale Study Group and TropNet members who collaborated in this study and FIND for providing the LAMP kits. We also thanks Terry N Illoh Villagrá for the English edition.

\section{Competing interests}

JC, AM, RP, GR y JR have no conflict of interest to declare. IG and XD are employees of FIND and co-developers of the malaria LAMP assay.

\section{Availability of data and materials}

All the raw data presented in this manuscript are archived and available as Supplementary files.

\section{Consent for publication}

All authors consent to publishing this article.

\section{Ethics approval and consent to participate}

This study was approved by the Ethics Committee of the Hospital Universitario Príncipe de Asturias (23/02/2016; Code Number: LIB 01/2016).

Consent to participate has been obtained from the patients.

\section{Funding}

This work received financial support from FIND with funds from the German Federal Ministry of Education and Research (BMBF) through the KfW Entwicklungsbank.

Received: 16 September 2016 Accepted: 22 December 2016

Published online: 07 January 2017

\section{References}

1. Hopkins H, Gonzalez IJ, Polley SD, Angutoko P, Ategeka J, Asiimwe C, et al. Highly sensitive detection of malaria parasitaemia in a malaria-endemic setting: performance of a new loop-mediated isothermal amplification kit in a remote clinic in Uganda. J Infect Dis. 2013;208:645-52.

2. Lucchi NW, Demas A, Narayanan J, Sumari D, Kabanywanyi A, Kachur SP, et al. Real-time fluorescence loop mediated isothermal amplification for the diagnosis of malaria. PLOS ONE. 2010:5:e13733.

3. Patel JC, Lucchi NW, Srivastava P, Lin JT, Sug-aram R, Aruncharus S, et al. Field evaluation of a real-time fluorescence loop-mediated isothermal amplification assay, realamp, for the diagnosis of malaria in Thailand and India. J Infect Dis. 2014;210:1180-7.

4. Yongkiettrakul S, Jaroenram W, Arunrut N, Chareanchim W, Pannengpetch S, Suebsing R, et al. Application of loop-mediated isothermal amplification assay combined with lateral flow dipstick for detection of Plasmodium falciparum and Plasmodium vivax. Parasitol Int. 2014;63:777-84.

5. Port JR, Nguetse C, Adukpo S, Velavan TP. A reliable and rapid method for molecular detection of malarial parasites using microwave irradiation and loop mediated isothermal amplification. Malar J. 2014;13:454.

6. Ramirez-Olivencia G, Rubio JM, Rivas P, Subirats M, Herrero MD, Lago M, et al. Imported submicroscopic malaria in Madrid. Malar J. 2012;11:324.

7. Rojo-Marcos G, Rubio-Munoz JM, Ramirez-Olivencia G, García-Bujalance S, Elcuaz-Romano R, Díaz-Menendez M, et al. Comparison of imported Plasmodium ovale curtisi and P. ovale wallikeri infections among patients in Spain, 2005-2011. Emerg Infect Dis. 2014;20:409-16.

8. Rubio JM, Benito A, Berzosa PJ, Roche J, Puente S, Subirats M, et al. Usefulness of seminested multiplex PCR in surveillance of imported malaria in Spain. J Clin Microbiol. 1999;37(10):3260-4.

9. Cuadros J, Perez-Tanoira R, Prieto-Perez L, Martin-Martin I, Berzosa P, González V, et al. Field evaluation of malaria microscopy, rapid malaria tests and loop-mediated isothermal amplification in a rural hospital in south western Ethiopia. PLOS ONE. 2015;10:e0142842.

10. Manual of standard operating procedures for malaria LAMP. http://www. finddiagnostics.org/export/sites/default/programs/malaria-afs/docs/ SOPS_LAMP_Malaria_AUG12.pdf. 2012.

11. Santiago Perez MI, Hervada Vidal X, Naveira Barbeito G, Silva LC, Fariñas $H$, Vázquez E, et al. The Epidat program. Rev Panam Salud Publica. 2010;27:80-2

12. Cotter C, Sturrock HJ, Hsiang MS, Liu J, Phillips AA, Hwang J, et al. The changing epidemiology of malaria elimination: new strategies for new challenges. Lancet. 2013;382:900-11.

13. Hopkins DR. Disease eradication. N Engl J Med. 2013;368:54-63.

14. Rojo-Marcos G, Cuadros-González J. Malaria y protozoos intestinales| Malaria and intestinal protozoa. Enferm Infecc Microbiol Clin. 2016;34:191-204.

15. Ocker R, Prompunjai $Y$, Chutipongvivate $S$, Karanis P. Malaria diagnosis by loop-mediated isothermal amplification (LAMP) in Thailand. Rev Inst Med Trop Sao Paulo. 2016;58:27.

16. Lau Y, Lai M, Fong M, Jelip J, Mahmud R. Loop-mediated isothermal amplification assay for identification of five human Plasmodium species in Malaysia. Am J Trop Med Hyg. 2015;94:336-9.

17. Morris U, Khamis M, Aydin-Schmidt B, Abass AK, Msellem MI, Nassor MH, et al. Field deployment of loop-mediated isothermal amplification for centralized mass-screening of asymptomatic malaria in Zanzibar: a preelimination setting. Malar J. 2015;14:205.

18. Lucchi NW, Ljolje D, Silva-Flannery L, Udhayakumar V. Use of malachite green-loop mediated isothermal amplification for detection of Plasmodium spp. parasites. PLoS ONE. 2016;11:e0151437. 